A. Iliev

Nagoya Math. J.

Vol. 132 (1993), 115-129

\title{
ALGEBRAIC THREEFOLDS WITH TWO EXTREMAL MORPHISMS
}

\author{
ATANAS ILIEV
}

\section{§. Introduction}

0.1. In [3] Mori gives a description of all extremal rays (extremal morphisms) arising on a smooth projective threefold with a numerically non-effective canonical bundle. Generally speaking, every smooth projective threefold $V$ with a numerically non-effective canonical class $K_{V}$ admits an extremal morphism $\pi: V \rightarrow Y$. The assumption that $V$ admits a non-trivial pair of extremal morphisms

$$
Y_{1} \stackrel{\pi_{1}}{\longleftarrow} V \stackrel{\pi_{2}}{\longrightarrow} Y_{2}
$$

imposes strong conditions on $V$. This is the essence of the Theorem 1.5 of the present work. In particular, we obtain a description of the threefolds which admit two biregular structures of conic bundles over non-singular surfaces $S_{1}=Y_{1}$ and $S_{2}=Y_{2}$. By the results of $\S 3$ the surfaces $S_{1}$ and $S_{2}$ must be either ruled surfaces with isomorphic basic curves, or $S_{1} \simeq S_{2} \simeq P^{2}$.

\subsection{Remarks}

0.2.1. In [5] E. Sato has obtained a description of the threefolds with two structures of $P^{1}$-bundles; this description corresponds to the Case A.a of Theorem 1.5. The second basic result of [5] states that if $\operatorname{dim} V \geqslant 3$ and $V$ admits two structures of projective space bundles over projective spaces $Y_{1}=P^{l}$ and $Y_{2}=$ $P^{m}$, then: either $V$ is a product $V=P^{l} \times P^{\mathrm{m}}$, or $l=m$ and $V=P\left(T_{P^{l}}\right)$.

0.2.2. Every Fano threefold $V$ with $\rho(V) \geqslant 2$ admits at least two extremal morphisms. However, in most of the cases $V$ admits a ray of the type $E_{1}$. Because of that, there are too many Fano threefolds with $\rho \geqslant 2$ in the list of Mori and Mukai in [4], in contrast to the list of Theorem 1.5 in which are classified only the strongly primitive ones.

Received July 26, 1989. 


\section{§1. Definitions and statement of the main theorem}

1.1. Everywhere in the present article, we suppose that the threefold $V$ is a smooth projective threefold over the field of complex numbers $\mathbf{C}$.

\subsection{Definitions}

1.2.1. $N V=\{1$-cycles on $V\} / \equiv \otimes \mathbf{R}$, where $\equiv$ denotes the numerical equivalence of cycles. $N V$ is a finite dimensional real vector space, which is dual to $N S(V) \otimes \mathbf{R}$, where $N S(V)$ is the Neron-Severi group of $V$.

1.2.2. (the Picard number of $V)=\rho(V)=\operatorname{dim}_{\mathbf{R}}(N V)$.

1.2.3. $\overline{N E}(V)$ is the closure of the convex cone $N E(V)$, generated by all the effective 1-cycles from $N V$ (in the metrical topology of the vector space $N V$ ).

1.2.4. Let $Z \in \overline{N E}(V)$. The half-line $R=\mathbf{R}_{+}[z]$ is called an extremal ray, if: (a) $-K_{V} . z>0$; (b) for all $Z_{1}, Z_{2} \in \overline{N E}(V)$, the assumption $Z_{1}+Z_{2} \in R$ implies $Z_{1} \in R$ and $Z_{2} \in R$, cf. [3].

1.2.5. Let $R$ be an extremal ray on $V$. Then, there exists a unique, up to an isomorphism, morphism $\pi: V \rightarrow Y$ corresponding to $R$, such that: (a) $\pi_{*} \mathscr{O}_{V}=\mathscr{O}_{Y}$; (b) if $C \subset V$ is an irreducible curve, then $[C] \in R$ if and only if $\operatorname{dim} \pi(C)=0$, cf. [3]. The morphism $\pi$ is called a contraction of the extremal ray $R$, or an extremal morphism (corresponding to $R$ ).

\subsection{Description of the extremal morphisms on $V$ (cf. [3])}

Let $\pi: V \rightarrow Y$ be an extremal morphism, and let $\rho(V) \geqslant 2$. Then $\pi$ can be one of the following:

\subsubsection{Type $E: \operatorname{dim} Y=3$}

The morphism $\pi$ is a contraction of a divisor $D$ on $V$, and $\pi$ corresponds to one of the types $E_{1}, E_{2}, E_{3}, E_{4}$, and $E_{5}$. In the case $E_{1}$ the morphism $\pi$ is a contraction of a ruled surface to a smooth curve, and the threefold $Y$ is smooth. In the case $E_{2}$ the morphism $\pi$ is a contraction of a divisor $D \simeq P^{2}$, with a normal bundle $\mathscr{O}_{D}(D) \simeq \mathscr{O}_{P^{2}}(-1)$, to a nonsingular point on $Y$. In the case $E_{3}$ the morphism $\pi$ is a contraction of a quadric $D \simeq P^{1} \times P^{1}$, with a normal bundle $\mathscr{O}(-1,-1)$, to an ordinary double point on $Y$. Moreover, the fibers $P^{1} \times t$ and $s \times P^{1}$ are numerically equivalent on $V$, for $t, s \in P^{1}$. In the case $E_{4}$ the morphism $\pi$ is a contraction of a quadratic cone $D \subset P^{3}$ to a double point on $Y$, and $\mathscr{O}_{D}(D) \simeq \mathscr{O}_{D}$ $\otimes \mathscr{O}_{P^{3}}(-1)$. In the case $E_{5}$ the morphism $\pi$ is a contraction of $D \simeq P^{2}$ to a quadruple point on $Y$, and $\mathscr{O}_{D}(D) \simeq \mathscr{O}_{P^{2}}(-2)$.

\subsubsection{Type $C: \operatorname{dim} Y=2$}

The variety $Y$ is a smooth projective surface, and $\pi$ corresponds to one of the types $C_{1}$ or $C_{2}$. In the case $C_{1}$ the morphism $\pi$ defines a conic bundle $\pi: V \rightarrow Y$; in the case $C_{2}$ the morphism $\pi$ defines a $P^{1}$-bundle $\pi: V \rightarrow Y$. 


\subsubsection{Type $D: \operatorname{dim} Y=1$}

The variety $Y$ is a smooth curve, $\rho(V)=\rho(Y)+1=2$, and $\pi$ corresponds to one of the types $D_{1}, D_{2}$, and $D_{3}$. In the case $D_{1}$ the threefold $V$ has a structure of a Del Pezzo bundle over the curve $Y$. In the case $D_{2}, V$ is isomorphic to a $P^{1} \times$ $P^{1}$-bundle over the curve $Y$. In the case $D_{3}$ the threefold $V$ is a $P^{2}$-bundle over $Y$.

1.4. Definition. The threefold $V$ is called strongly primitive if there are no extremal rays of type $E_{1}$ on $V$.

\subsection{The Main Theorem}

THEOREM. Let $V$ be a (smooth, projective) strongly primitive threefold which admits two extremal morphisms $\pi_{1}: V \rightarrow Y_{1}$ and $\pi_{2}: V \rightarrow Y_{2}$. Then, the following cases are possible:

Case 1. The morphisms $\pi_{1}$ and $\pi_{2}$ correspond to the type $C$. Then $2 \leqslant \rho(V) \leqslant 3$ and:

(1.A) If $\rho(V)=3$, then

either: (A.a). $V \simeq S_{1} \times{ }_{C} S_{2}$, where $S_{1}$ and $S_{2}$ are ruled surfaces over a curve $C$,

or: (A.b). $V$ is a two-sheeted covering of $S_{1} \times{ }_{c} S_{2}$, where $S_{1}, S_{2}$, and $C$ are as in (A.a).

(1.B) If $\rho(V)=2$, then $V$ is a Fano threefold (see Corollary 2.6.2).

Case 2. The morphism $\pi_{1}$ corresponds to the type $C$, and the morphism $\pi_{2}$ corresponds to one of the types $D$ or $E$. Then $V$ is a Fano threefold (see Corollary 4.2, Corollaries 5.3 and 5.4).

Case 3. Let the extremal morphisms $\pi_{1}, \pi_{2}, \ldots$ on $V$ be of the type $E$. Then the corresponding divisors $D_{1}, D_{2}, \ldots$ are mutually disjoint (see $\S 7$ ).

Remark. The rest of the paper is devoted to the proof of Theorem 1.5. Especially, Case 1 is discussed in $\S 2$ and $\S 3$. It follows from the considerations in $\S 3$ that the double covering $\pi: V \rightarrow S_{1} \times{ }_{C} S_{2}$, in case (A.b), has the following properties:

Let $\mathscr{E}_{i}$ be a normalized locally free sheaf of rank 2, over the base curve $C$, such that $S_{i}=P_{C}\left(\mathscr{E}_{i}\right), i=1,2($ see $[1, \mathrm{ch} . \mathrm{V}, \S 2])$. Let $e_{i}=-\operatorname{deg}\left(\operatorname{det} \mathscr{E}_{i}\right)$, let $\varphi_{i}$ be the general fibre of $S_{i} \rightarrow C$, and let $b_{i}$ be the section of $S_{i}$ such that $\mathscr{L}\left(b_{i}\right)=$ $\mathscr{O}_{P\left(\mathscr{E}_{i}\right)}(1), i=1,2$. Let $p_{i}: S_{1} \times{ }_{C} S_{2} \rightarrow S_{i}$ be the natural projections, and let $C_{i}=$ $p_{i}^{*}\left(b_{i}\right), F_{i}=p_{i}^{*}\left(\varphi_{i}\right), i=1,2$. Then $F_{1}$ and $F_{2}$ are numerically equivalent, i.e. $F_{1} \equiv F_{2} \equiv F$ for some $F \in p_{1}^{*}\left(\operatorname{Pic} S_{1}\right) \cap p_{2}^{*}$ (Pic $S_{2}$ ). The branch divisor $B \subset S_{1}$ $\times{ }_{C} S_{2}$ of $\pi$ is smooth, and $B$ is numerically equivalent to $2 . C_{1}+2 . C_{2}+2 q . F$ for some $q>0$. Moreover, the threefold $V$ is a standard conic bundle over $S_{i}$ with a 
discriminant curve $\Delta_{i} \equiv 4 . b_{i}+\left(4 q-2 e_{i}\right) \cdot \varphi_{i}$, where $\{i, j\}=\{1,2\}$.

\section{§2. The case $(C, C)$}

2.1. Let $\pi_{1}$ and $\pi_{2}$ be of type $C$. Let $\pi_{1}: V \rightarrow Y_{1}$ and $\pi_{2}: V \rightarrow Y_{2}$ be the corresponding extremal morphisms. In particular, $S_{1}=Y_{1}$ and $S_{2}=Y_{2}$ are smooth surfaces (see 1.3.2). Denote by $f_{k}$ the general fiber of the morphism $\pi_{k}, k=1,2$.

2.2. Proposition. If $\rho(V) \geqslant 3$, then $\rho(V)=3$, and $S_{1}$ and $S_{2}$ are ruled surfaces.

Proof.

2.2.1. Let $H$ be a very ample divisor on $S_{2}$, and let $C \in|H|$ be a smooth curve. Then $\left(\pi_{2}^{*} C, \pi_{2}^{*} C\right)_{V}=m . f_{2}$, where $m=(C, C)_{S_{2}}>0$. Therefore, $\pi_{2}^{*} C \notin$ $\pi_{1}^{*}\left(\right.$ Pic $\left.S_{1}\right)$; hence, the mapping $\pi_{1}: \pi_{2}^{*} C \rightarrow S_{1}$ is surjective. Since $\mathfrak{x}\left(\pi_{2}^{*} C\right)=-\infty$, then $\mathfrak{x}\left(S_{1}\right)=-\infty$ (here $\mathfrak{x}(X)$ is the Kodaira dimension of $X$ ). Similarly $\mathfrak{x}\left(S_{2}\right)=$ $-\infty$. Consequently, there exist morphisms $h_{k}: S_{k} \rightarrow S_{k, o}$, where $S_{k, o}$ are ruled surfaces or $P^{2}$. As $\rho(V) \geqslant 3$, then $\rho\left(S_{k}\right) \geqslant 2$.

Let, for example, $S_{1,0}=P^{2}$. Then the surface $S_{1}$ is rational, and the morphism $h_{1}: S_{1} \rightarrow S_{1, o}=P^{2}$ is non-trivial; in the opposite case $\rho(V)=\rho\left(P^{2}\right)+1=2$, which contradicts the assumption $\rho(V) \geqslant 3$. Consequently, there exists a morphism $h_{1}^{\prime}: S_{1} \rightarrow \mathbf{F}_{1}$, such that $h_{1}=h_{1}^{\prime} . \sigma$, where $\sigma: \boldsymbol{F}_{1} \rightarrow P^{2}$ is a blowing-up of a point in $P^{2}$. Therefore, we can always assume that $S_{1, o}$ and $S_{2, o}$ are ruled surfaces (rational or non-rational).

Let $S_{k, o}=P\left(\mathscr{E}_{k}\right)$, let $\mathscr{L}\left(b_{k, o}\right)=\mathscr{O}_{P\left(\mathscr{E}_{K}\right)}(1)$, and let $\varphi_{k, o}$ be the general fiber of $S_{k, o}, k=1,2$ (see the Remark after Theorem 1.5). Let

$$
\text { Num } S_{k}=\mathbf{Z} b_{k} \oplus \mathbf{Z} \varphi_{k} \oplus \bigoplus_{i=1}^{m_{k}} \mathbf{Z}_{\varepsilon_{k, i}},
$$

where $\varepsilon_{k, i}$ are the exceptional curves of $h_{k}$, and $b_{k}$ and $\varphi_{k}$ are the preimages of $b_{k, o}$ and $\varphi_{k, o}$ on $S_{k}, k=1,2$. Let $m=\rho(V)-1$. Obviously $\rho\left(S_{k}\right)=m=m_{k}+2$, $k=1,2$.

Let $C_{1}=\pi_{1}^{*} b_{1}, C_{2}=\pi_{1}^{*} \varphi_{1}, C_{i+2}=\pi_{1}^{*} \varepsilon_{1, i}, D_{1}=\pi_{2}^{*} b_{2}, D_{2}=\pi_{2}^{*} \varphi_{2}, D_{i+2}=$ $\pi_{2}^{*} \varepsilon_{2, i}(i=1,2, \ldots m-2)$. If $\pi_{k}: V \rightarrow S_{k}$ is a conic bundle, then Pic $V=$ $\pi_{2}^{*}$ Pic $S_{k}+\mathbf{Z} K_{V}$; if $\pi_{k}: V \rightarrow S_{k}$ is a $P^{1}$-bundle, then Pic $V=\pi_{k}^{*} \operatorname{Pic} S_{k}+\mathbf{Z} L_{k}$, where $L_{k}$ corresponds to a section of $\pi_{k}$. In both cases

$$
\text { 2. Pic } V \subseteq \pi_{k}^{*} \operatorname{Pic} S_{k}+\mathbf{Z} K_{V} \text {, }
$$

i.e. the divisors $D_{i}$ (resp. $C_{i}$ ) are linear combinations, with integer or half-integer 
coefficients, of the divisors $C_{i}$ (resp. $D_{i}$ ) and $K_{V}$ (in the numerical sense). Therefore, there exists a system of equations of the form:

$$
\begin{aligned}
& C_{i}+\sum_{j} d_{i j} D_{j} \equiv-d_{i} K_{V} \\
& \sum_{j} c_{i j} C_{j}+D_{i} \equiv-c_{i} K_{V},
\end{aligned}
$$

where the numbers $2 d_{i j}, 2 d_{i}, 2 c_{i j}$, and $2 c_{i}$ are integer.

Let $D=\left(d_{i j}\right), C=\left(c_{i j}\right), d=\left(d_{1}, \ldots, d_{m}\right)^{t}, c=\left(c_{1}, \ldots, c_{m}\right)^{t}$, and let $E$ be the unit matrix of rank $m$. By the adjunction formula $K_{V} \cdot f_{k}=-2, k=1,2$; and from 2.2 .2 we conclude that $C_{i} . f_{2}=2 d_{i}, D_{i} . f_{1}=2 c_{i}$. The integers $2 d_{i}$ and $2 c_{i}$ are non-negative; they can be interpreted as follows:

If $d_{i}=0$, then $C_{i} \in \pi_{2}^{*} \mathrm{Pic} S_{2}$;

if $d_{i}>0$, then $d_{i}=$ the degree of the covering $\pi_{2}: C_{i} \rightarrow S_{2}$; (similarly - for $c_{i}$ ). Further, from 2.2 .2 we derive:

$\left(-c_{i}+\sum_{l} c_{i l} d_{l}\right) \cdot K_{V} \equiv D_{i}-\sum_{l, j} c_{i l} d_{l j} D_{j}, i=1,2, \ldots . m$. Therefore, from the formula connecting Pic $V$ and Pic $S_{2}$, we obtain that the both sides of the last equation are equal to zero, in the numerical sense. Hence, $C . D=E$, and $C d=c$. These matrix equations will be used in the proof of Proposition 2.3.

2.2.3. Let $C_{i} C_{j}=\gamma_{i j} f_{1}, D_{i} D_{j}=\delta_{i j} f_{2}, k_{i j}=K_{V} C_{i} D_{j}$. After multiplying the first $m$ equations from 2.2 .2 by $C_{i} D_{j}$ we obtain the following system:

2.2.4. $R_{i j k}=2 d_{i} \sum_{l} d_{k l} \delta_{l j}+d_{k} k_{i j}+\gamma_{k i} .2 c_{j}=0$.

By the choice of the curves $b_{k}, \varphi_{k}, \varepsilon_{k i}$, the numbers $\gamma_{k i}$ and $\delta_{l j}$ satisfy the following conditions:

2.2.5. (a) $\gamma_{i i}=-p_{i}<0, \delta_{i i}=-q_{i}<0, i \geqslant 3$;

(b) $\gamma_{1 i}=\gamma_{2 i}=\delta_{1 i}=\delta_{2 i}=0, i \geqslant 3$;

(c) $\gamma_{22}=\delta_{22}=0, \gamma_{11}=-e_{1}, \delta_{11}=-e_{2}$, where $-e_{k}=\left(b_{k}, b_{k}\right)_{s_{k}}=\left(b_{k, o}, b_{k, o}\right)_{s_{k, o}}, k=1,2$.

2.2.6. Lemma. If $d_{2}=0$, then $d_{3}=\cdots=d_{m}=0$ (similarly - for $\left.c_{i}\right)$.

Proof. Every $\varepsilon_{1, i}$ is a component of some degenerating fiber $\varphi_{1, i} \equiv \varphi_{1}$ of $h_{1}$, $\varphi_{1, i}$ being a linear combination with integer coefficients of exceptional curves and the preimage of some fiber of $S_{1, o}$. Let, for example, $\varphi_{1} \equiv \sum_{n \geqslant 0} \lambda_{n} \varepsilon_{1, n}$, where $\lambda_{n} \geqslant 0$ and $\varepsilon_{1, o}$ is the proper preimage of some fiber of $S_{1, o}$, over which we take blowing-ups. Then

$$
\text { 2.2.7. } 0=2 d_{2}=C_{2} f_{2}=\lambda_{0} . \pi_{1}^{*} \varepsilon_{1,0} f_{2}+\sum_{n \geqslant 1} \lambda_{n} .2 d_{n+2} .
$$

Hence, $2 d_{i+2}=C_{i+2} f_{2}=\pi_{1}^{*} \varepsilon_{1, i} f_{2}=0$. 
2.2.8. Lemma. If $m=\rho(V)-1 \geqslant 3$, then $\Pi_{i=3}^{m} c_{i} d_{i}=0$.

Let us look at the equations $R_{13 k}=0, k \geqslant 2$, and $R_{23 k}=0, k \geqslant 2$ (see 2.2.4). We shall give a proof of 2.2 .8 on an example, which is not different from the general case.

Example. $m=4$; i.e. from 2.2 .5 we have $\delta_{33} \neq 0, \delta_{43} \neq 0, \delta_{13}=\delta_{23}=0$. For definiteness, we may assume that $\delta_{33}=-2$ and $\delta_{43}=1$; therefore $\delta_{44}=-1$. The surface $S_{2}$ is obtained from $S_{2, o}$ after blowing-up a point not lying on the base section, and a second blowing-up with a centre lying on the first exceptional divisor. The equations $R_{13 k}=0$ and $R_{23 k}=0, k \geqslant 2$, take the form:

$$
\begin{array}{ll}
\text { 2.2.9. } & R_{132}=-2 c_{3}+\left(-2 d_{23}+d_{24}\right) \cdot 2 d_{1}=-d_{2} k_{13} \\
& R_{13 k}=\left(-2 d_{k 3}+d_{k 4}\right) \cdot 2 d_{1}=-d_{k} k_{13}, k=3,4 \\
& R_{23 k}=\left(-2 d_{k 3}+d_{k 4}\right) \cdot 2 d_{2}=-d_{k} k_{23}, k=2,3,4
\end{array}
$$

From 2.2.9 we easily derive that either $d_{2}=0$ (and hence, according to Lemma 2.2.6, $d_{3}=\cdots=d_{m}=0$ ), or the assumption $d_{3} \neq 0$ implies $c_{3}=0$.

\subsubsection{Lemma. If $\rho(V) \geqslant 3$, then $\rho(V)=3$.}

Proof. According to Lemma 2.2.8, if $m \geqslant 3$, then $\Pi_{i \geqslant 3} c_{i} d_{i}=0$. Let, for example, $c_{3}=0$. Then $D_{3} \in \pi_{1}^{*}$ Pic $S_{1}$. Hence $D_{3}=\pi_{1}^{*} C$, where $C \in$ Pic $S_{1}$ and $(C, C)_{S_{1}}=r \in \mathbf{Z}$. Then $-q_{3} f_{2}=\left(D_{3}, D_{3}\right)_{V}=\left(\pi_{1}^{*} C, \pi_{1}^{*} C\right)_{V}=r f_{1}$, where $q_{3}>0$ (i.e. $\left.q_{3} \neq 0\right)-$ a contradiction. Therefore $m=\rho(V)-1=2$, and the Proposition 2.2 is proved.

2.3. Proposition. Let $\rho(V)=3$. Then $\pi_{1}^{*} \varphi_{1} \in \pi_{2}^{*} \operatorname{Pic} S_{2}$ and $\pi_{2}^{*} \varphi_{2} \in$ $\pi_{1}^{*}$ Pic $S_{1}$, where $\varphi_{k}$ is the general fiber of the ruled surface $S_{k}, k=1,2$.

Proof. For convenience, we shall change the notation. As $m=2$, the system 2.2.2 takes the form:

$$
\text { 2.3.1. } \begin{aligned}
-C_{1}+g_{1} C_{2}+d_{1} F_{2} & \equiv r_{1} K_{V} \\
-F_{1}+b_{1} C_{2}+a_{1} F_{2} & \equiv c_{1} K_{V} \\
g_{2} C_{1}+d_{2} F_{1}-C_{2} & \equiv r_{2} K_{V} \\
b_{2} C_{1}+a_{2} F_{1} & -F_{2} \equiv c_{2} K_{V},
\end{aligned}
$$

where $F_{k}=\pi_{k}^{*} \varphi_{k}, C_{k}=\pi_{k}^{*} b_{k}, k=1,2$, and all the coefficients are either integers, or half-integers.

The equality $C . D=E$ (see 2.2.2) implies $g_{2}=\varepsilon a_{1}, d_{2}=-\varepsilon d_{1}, b_{2}=-\varepsilon b_{1}$, 
and $a_{2}=\varepsilon g_{1}$, where $\varepsilon=\left(g_{1} a_{1}-b_{1} d_{1}\right)^{-1}$. From $C d=c$ and $D c=d$ (ibid.) we obtain:

2.3.2.

$$
\begin{aligned}
& c_{2}+a_{2} c_{1}+b_{2} r_{1}=0 \\
& r_{2}+d_{2} c_{1}+g_{2} r_{1}=0 \\
& c_{1}+a_{1} c_{2}+b_{1} r_{2}=0 \\
& r_{1}+d_{1} c_{2}+g_{1} r_{2}=0 .
\end{aligned}
$$

After multiplying both sides of the equalities 2.3 .1 by $f_{1}$ and $f_{2}$ we obtain $F_{1} f_{2}=$ $2 c_{1}, F_{2} f_{1}=2 c_{2}, C_{1} f_{2}=2 r_{1}$, and $C_{2} f_{1}=2 r_{2}$. The system 2.2 .5 for $\gamma_{i j}$ and $\delta_{i j}$ takes the form:

$$
\begin{aligned}
& C_{1}^{2}=-e_{1} f_{1}, C_{2}^{2}=-e_{2} f_{2}, C_{1} F_{1}=f_{1}, \\
& C_{2} F_{2}=f_{2}, F_{1}^{2}=F_{2}^{2}=0 .
\end{aligned}
$$

We divide the proof in several cases:

Case 1. $S_{1}$ and $S_{2}$ are rational.

Claim. In Case 1 , the equality $c_{1} c_{2} r_{1} r_{2}=0$ is fulfilled.

Proof of the Claim. Assume that $c_{1} c_{2} r_{1} r_{2} \neq 0$; and let $\varepsilon<0$. From the equation $c_{1} K_{V} F_{1} C_{2}=2 a_{1} c_{1}-2 b_{1} e_{2} c_{1}$, we get $K_{V} F_{1} C_{2}=2 a_{1}-2 b_{1} e_{2}$; therefore $c_{2}\left(2 a_{1}-2 b_{1} e_{2}\right)=c_{2} K_{V} F_{1} C_{2}=-2 c_{1}+2 b_{2} r_{2}$. By 2.3.2, $2 c_{1}+2 a_{1} c_{2}=-2 b_{1} r_{2}$, where $b_{2}=-\varepsilon b_{1}$. Hence:

$$
\text { 2.3.3. }(2 \varepsilon-2) b_{1} r_{2}=2 b_{1} e_{2} c_{2} .
$$

From $\varepsilon<0, r_{2}>0, c_{2}>0$, and $e_{2} \geqslant 0$, we get that $b_{1}=0$; in particular $b_{2}=$ $-\varepsilon b_{1}=0$. Thus, from $c_{1} K_{V} F_{1} F_{2}=2 b_{1} c_{1}$ and $c_{1}>0$, we obtain that $K_{V} F_{1} F_{2}=0$. Then, from $r_{2} K_{V} F_{1} F_{2}=0$ and $r_{1} K_{V} F_{1} F_{2}=0$, we conclude that $-2 c_{1}+2 g_{2} c_{2}=$ 0 and $-2 c_{2}+2 g_{1} c_{1}=0$. Therefore $g_{1}>0, g_{2}>0$, and $g_{1} \cdot g_{2}=1$.

From $K_{V} F_{1} C_{2}=2 a_{1}$, and from the equations of the type $R_{i j k}=0$ for $r_{2} K_{V} F_{1} C_{2}$ we obtain:

$$
\text { 2.3.4. }\left(a_{1}-g_{2}\right) r_{2}=2 e_{2} c_{1},
$$

where $a_{1}=\varepsilon g_{1}, g_{2}=\varepsilon a_{1}, g_{1}>0, g_{2}>0$, and $\varepsilon<0$. In that case, the equation 2.3.4 contradicts the assumption that $c_{1}>0$ and $r_{2}>0$.

Let $\varepsilon<0$ and $c_{1} c_{2} r_{1} r_{2}=0$. In particular, if $r_{1}=1$ then $2 e_{1} c_{2}=r_{1} K_{V} F_{2} C_{1}=0$. Therefore, either $c_{2}=0$, or $e_{1}=0$. If $e_{1}=0$, then $S_{1} \simeq P^{1} \times P^{1}$, and we can assume that $b_{1} \subset S_{1}$ is a fiber (cf. 2.2.1).

Let $c_{1}=0$, but $c_{2}>0$. Then 2.3 .2 implies that $c_{2}+b_{2} r_{1}=0$, i.e. $b_{2}<0$. But, 
from the equations $0=c_{1} K_{V} F_{2} C_{1}=-2 c_{2}+2 b_{1} r_{1}$ and $b_{2}=-\varepsilon b_{1}, \varepsilon<0$, we obtain that $b_{2}>0$, which is impossible.

Corollary. If $\varepsilon<0$, and $S_{1}$ and $S_{2}$ are rational, then $c_{1}=c_{2}=0$.

If $\varepsilon>0$, we come to a contradiction in the same way. The Claim is proved. Proceeding in a similar way, from the above Claim and from 2.3.2, we obtain the following

Corollary. In the Case 1 we have $c_{1}=c_{2}=0$.

Then, from 2.3.1, we obtain that $F_{1} \in \pi_{2}^{*} \operatorname{Pic} S_{2}$ and $F_{2} \in \pi_{1}^{*} \operatorname{Pic} S_{1}$.

Case 2. $S_{1}$ or $S_{2}$ is non-rational.

Let, for example, $S_{1}$ be an irregular ruled surface and let $g_{1}: S_{1} \rightarrow C, g_{2}: S_{2}$ $\rightarrow C^{\prime}$ be the corresponding representations of the surfaces $S_{1}$ and $S_{2}$ as $P^{1}$-bundles over the curves $C$ and $C^{\prime}$, where $g(C)=g \geqslant 1$. Then the general fibers of $\left|L_{1}\right|=g_{1} \pi_{1}: V \rightarrow C$ and $\left|L_{2}\right|=g_{2} \pi_{2}: V \rightarrow C^{\prime}$ are rational surfaces.

Let $\left|L_{1}\right| \neq\left|L_{2}\right|$. Then $f=\left.L_{2}\right|_{L_{1}}$ is a curve on $L_{1}$ and $(f, f)_{L_{1}}=L_{2} L_{2} L_{1}=0$. Hence, the restriction $\left.\left|L_{2}\right|\right|_{L_{1}}: L_{1} \rightarrow C$ defines, on the rational surface $L_{1}$, a structure of bundle with rational curves as fibers and with a non-rational base $C$, which is impossible. Therefore $C^{\prime} \simeq C$, and the diagram

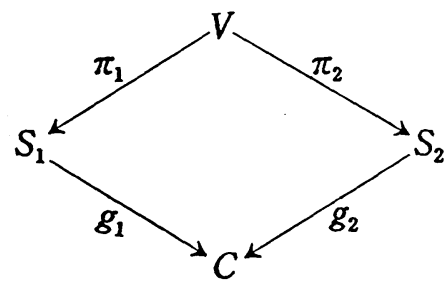

where $g(C)=g \geqslant 1$, is commutative. Evidently, in this case $\pi_{1}^{*} \varphi_{1} \in \pi_{2}^{*} \operatorname{Pic} S_{2}$ and $\pi_{2}^{*} \varphi_{2} \in \pi_{1}^{*}$ Pic $S_{1}$. The Proposition 2.3 is proved.

2.4. Corollary. If $\rho(V)=3$, then the equation 2.3 .2 take the form:

$$
r K_{V} \equiv-C_{1}-C_{2}+d F,
$$

where $F_{1} \equiv F_{2} \equiv F \in \pi_{1}^{*}$ Pic $S_{1} \cap \pi_{2}^{*}$ Pic $S_{2}$, the numbers $2 r$ and $2 d$ are integer, and $r>0$. 
The Corollary is a direct consequence from Proposition 2.3, and from the first and the third equations of 2.3.1. Note that from the two other equations of 2.3.1 we obtain that $b_{1}=b_{2}=0$ and $a_{1}=a_{2}=1$. Then, the former two equations give $\varepsilon=-1$.

2.5. Corollary. If $\rho(V)=3$, then there exists a curve $C$ such that the diagram

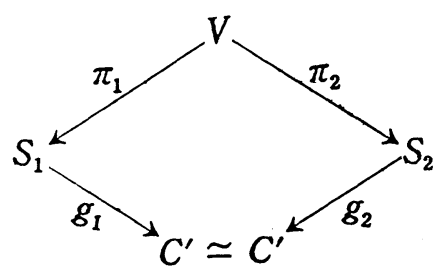

is commutative.

Proof. For $S_{1}$ and $S_{2}$ - non-rational, the Corollary is proved in 2.3, Case 2 .

Let $S_{1}$ and $S_{2}$ be rational ruled surfaces. By Proposition 2.3, we have $\pi_{1}^{*} \varphi_{1}$ $\in \pi_{2}^{*} \operatorname{Pic} S_{2}$ and $\pi_{2}^{*} \varphi_{2} \in \pi_{1}^{*}$ Pic $S_{1}$. Consequently, there are correctly defined morphisms $\lambda=g_{1} \pi_{1} \pi_{2}^{-1} g_{2}^{-1}: C^{\prime} \rightarrow C$ and $\lambda^{-1}=g_{2} \pi_{2} \pi_{1}^{-1} g_{1}^{-1}: C \rightarrow C^{\prime}$, where $g_{1}: S_{1} \rightarrow C \simeq P^{1}$ and $g_{2}: S_{2} \rightarrow C^{\prime} \simeq P^{1}$ define structures of ruled surfaces on $S_{1}$ and $S_{2}$. Therefore $\lambda \in$ Aut $P^{1}$; and if we replace $g_{2}$ by $\lambda . g_{2}$, we shall obtain the commutative diagram from above.

\subsection{Case $\rho(V)=2$}

Let us consider the case $\rho(V)<3$. Then $\rho(V)=2$, and there are on $V$ two extremal rays $R_{1}$ and $R_{2}$ of type $C$. As $\rho(V)=\operatorname{dim}_{\mathbf{R}}(N V)=2$, then $R_{1}$ and $R_{2}$ form a base of the two-dimensional real vector space $N V$. Let $R_{1}=\mathbf{R}_{+}\left[l_{1}\right]$ and $R_{2}$ $=\mathbf{R}_{+}\left[l_{2}\right]$. Since $R_{1}$ and $R_{2}$ are extremal rays in the two-dimensional cone $\overline{N E}(V) \subset N V$, and since $K_{V} \cdot l_{1}<0, K_{V} \cdot l_{2}<0$, then $K_{V} . Z<0$ for any $Z \in$ $\overline{N E}(V)$. By the Kleiman's criterion we derive that $-K_{V}$ is ample, i.e. $V$ is a Fano threefold.

2.6.1. Corollary. If $\rho(V)=2$ and $\left(\pi_{1}, \pi_{2}\right)$ is of type $(C, C)$, then $V$ is a Fano threefold.

2.6.2. Corollary (see [4]). In the conditions of 2.6.1, the threefold $V$ is one of the following: 
(1) a divisor of bidegree $(2,2)$ in $P^{2} \times P^{2}$;

(2) a divisor of bidegree $(2,1)$ in $P^{2} \times P^{2}$;

(3) a divisor of bidegree $(1,1)$ in $P^{2} \times P^{2}$;

(4) a two-sheeted covering of a divisor $D$ of bidegree $(1,1)$ in $P^{2} \times P^{2}$, with a branch divisor $B \in\left|-K_{D}\right|$.

\$3. Construction of threefolds of type $(C, C)$ with $\rho=3$,

Let $V$ be of type $(C, C)$, and let $\pi_{1}: V \rightarrow S_{1}, \pi_{2}: V \rightarrow S_{2}, C$, etc., be as in $\S 2$. It follows from the considerations in $\S 2$ that there exists a commutative diagram of natural morphisms:

3.1.

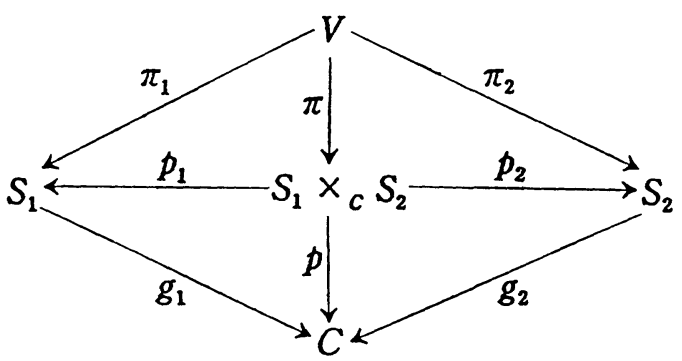

where $p_{1}$ and $p_{2}$ are the natural projections, and $\operatorname{deg} \pi=1$ or $\operatorname{deg} \pi=2$ (see 2.5).

We shall consider the case $\operatorname{deg} \pi=2$. In this setting, we shall obtain numerical formulae for the branch divisor of the double covering $\pi$.

3.2. Let $f_{k}$ be the (general) fiber of $\pi_{k}$, and let $\sigma_{k}$ be the (general) fiber of $R_{k}$, $k=1,2$. Evidently $\sigma_{k} \simeq P^{1}$ for any $\sigma_{k}, k=1,2$. Let $\mathscr{E}_{k}, b_{k}, \varphi_{k}, C_{k}, k=1,2$, and $F_{1} \equiv F_{2} \equiv F$ be as in the Remark after Theorem 1.5. Let $\varepsilon_{k}=\operatorname{det}\left(\mathscr{E}_{k}\right), e_{k}=$ - $\operatorname{deg}\left(\varepsilon_{k}\right)$; and let $C_{1 V}=\pi^{*} C_{1}, C_{2 V}=\pi^{*} C_{2}, F_{1 V} \equiv F_{2 V} \equiv F_{V}$ be the divisors on $V$, in the sense indicated in Corollary 2.4, i.e. $F_{V} \equiv \pi^{*} p^{*}(x) \equiv \pi^{*} F$ (where $F \equiv$ $\left.p^{*}(x), \quad x \in C\right)$. It is easy to see that:

3.3. $K_{S_{1} \times{ }_{C} S_{2}}=-2 C_{1}-2 C_{2}+\left(\varepsilon_{1}+\varepsilon_{2}+k\right) F$, where $k=K_{C}$ is the canonical divisor of $C$. The branch divisor $B$ of $\pi$ has the form:

3.4. $B=2 q_{1} C_{1}+2 q_{2} C_{2}+2 q F$, where $q_{1}$ and $q_{2}$ are integers, and $2 q F$ is used in the sense that $2 q F=p^{*}(q)$ for the divisor $q$ on $C$.

We claim that $q_{1}=q_{2}=1$.

In fact, as $p_{1}: S_{1} \times{ }_{C} S_{2} \rightarrow S_{1}$ is a $P^{1}$-bundle, then $\operatorname{Pic}\left(S_{1} \times{ }_{c} S_{2}\right)=p_{1}^{*} \operatorname{Pic} S_{1}$ $\oplus \mathbf{Z} C_{2}$ (since $C_{2}$ is an 1 -section of $p_{1}$ ). Therefore $\operatorname{Pic}\left(S_{1} \times{ }_{C} S_{2}\right)=\mathbf{Z} C_{1} \oplus g_{1}^{*}$ 
$(\operatorname{Pic} C) \oplus \mathbf{Z} C_{2}$, in sense that $g_{1}^{*}(\operatorname{Pic} C) . F=p_{1}^{*} g_{1}^{*}(\operatorname{Pic} C)=p^{*}(\operatorname{Pic} C)$. As $\pi: f_{1} \rightarrow \sigma_{1}$ is a two-sheeted covering for the general $f_{1} \simeq P^{1}$ and $\pi\left(f_{1}\right)=\sigma_{1} \simeq P^{1}$, then it has two branch points. Therefore $\operatorname{deg}\left(\left.B\right|_{\sigma_{1}}\right)=\operatorname{deg}\left(\left.B\right|_{\sigma_{2}}\right)=2$, i.e. $2=$ $\operatorname{deg}\left(\left.B\right|_{\sigma_{1}}\right)=\left(2 q_{1} C_{1}+2 q_{2} C_{2}+2 q F\right) \cdot \sigma_{1}=2 q_{2} C_{2} \sigma_{1}=2 q_{2}$; similarly - for $q_{1}$. As $V$ is smooth, then $B$ is smooth, and we derive:

COROLlary. The (smooth) branch divisor $B$ of $\pi$ has the form

$$
B=2 C_{1}+2 C_{2}+2 q F
$$

for some divisor $\mathfrak{q}$ on $C$, where $2 \mathfrak{q} F=p^{*}(2 \mathfrak{q})$.

3.5. In the context of the situation, we shall derive some necessary numerical conditions for $B$.

The general surface $C_{1 V}=\pi^{*} C_{1}$ is smooth, and it is a two-sheeted covering of $C_{1}$ with a branch divisor $\left.B\right|_{C_{1}}=\left.\left(2 C_{1}+2 C_{2}+2 q F\right)\right|_{C_{1}}=2 C_{12}+2(\varepsilon+q) \sigma_{1}$, where $C_{12}=C_{1} . C_{2}$, and $\left(C_{12}, C_{12}\right)_{C_{1}}=C_{2} C_{2} C_{1}=-e_{2} \sigma_{2} C_{1}=-e_{2}$. Therefore, for the existence of a (smooth) effective divisor $C_{12} \subset C_{1}$, one must have $2(q-$ $\left.e_{1}\right) \geqslant 2 e_{2}$ if $e_{2} \geqslant 0$, or $2\left(q-e_{1}\right) \geqslant \frac{1}{2} \cdot 2 e_{2}=e_{2}$ if $e_{2}<0$ (see [1, Ch. V, §2]); here $q=\operatorname{deg}(q)$. The same restrictions are available for $C_{2}$ and $e_{1}$, and we derive:

Corollary. Let $B, e_{1}$, and $e_{2}$ be as in $3.2-3.4$. Then for $q=\operatorname{deg}(q)$ we have:

(a) $q \geqslant e_{1}+e_{2}$ if $e_{1} \geqslant 0, e_{2} \geqslant 0$;

(b) $2 q \geqslant 2 e_{1}+e_{2}$ if $e_{1} \geqslant 0, e_{2}<0$;

(c) $2 q \geqslant e_{1}+2 e_{2}$ if $e_{1}<0, e_{2} \geqslant 0$;

(d) $2 q \geqslant \max \left\{2 e_{1}+e_{2}, e_{1}+2 e_{2}\right\}$ if $e_{1}<0, e_{2}<0$.

3.6. The canonical divisor $K_{V}$, and the surfaces $C_{1 V}$ and $C_{2 V}$

3.6.1. It follows from the preceding that

$K_{V}=-C_{1 V}-C_{2 V}+\left(\varepsilon_{1}+\varepsilon_{2}+\mathfrak{q}\right) F_{V}$. Therefore, by the adjunction formula

$K_{C_{1 V}}=-C_{12 V}-\left(\varepsilon_{1}+\varepsilon_{2}+\mathfrak{q}+\mathfrak{q}\right) f_{1}$, where $C_{12 V}=C_{1 V} \cdot C_{2 V}$. Evidently, the self-intersection number of $C_{12 V}$ in $C_{1 V}$ is equal to $-2 e_{2}$, and $C_{12 V} \cdot f_{1}=2$. Therefore $K_{C_{1 V}} \cdot K_{C_{1 V}}=(8-8 g)-\left(4 q-4 e_{1}-2 e_{2}\right)$, where $q=\operatorname{deg}(q)$ and $g=g(C)$.

3.6.2. From the Corollary in 3.5 , we obtain $4 q-2 e_{1}-2 e_{2} \geqslant 0$; similarly for $C_{2 V}$. From $K_{C_{1 V}} \equiv-C_{2 V} l_{C_{1 V}}+\left(2 g-2-e_{1}-e_{2}+q\right)$. $f_{1}$ we conclude that 
$h_{1}: C_{1 V} \rightarrow\left(C_{1 V}\right)_{\min }$ is a composition of $\sigma$-processes with centers lying on the curve $h_{1}\left(C_{12 V}\right)$; here $\left(C_{1 V}\right)_{\min }$ is some (relatively) minimal model of $C_{1 V}$.

3.6.3. For $F_{V}=\pi^{*} p^{*}(x), x \in C$, we have similarly: $K_{F_{V}}=\left(-C_{1 V}-\right.$ $\left.C_{2 V}\right) . F_{V}$ and $K_{F_{V}} \cdot K_{F_{V}}=4$. The surface $F_{V}$ is obtained from $P^{2}$ after blowing-up of five points.

\subsection{Examples of Fano threefolds of type $(C, C)$}

3.7.1. Let $\operatorname{deg} \pi=2$. Then $V$ is of type $\left(C_{1}, C_{1}\right)$, and $K_{V}=-C_{1 V}-C_{2 V}$ $+\left(2 g-2+q-e_{1}-e_{2}\right) . F_{V}$. Let, moreover, $V$ be a Fano threefold. Then $K_{V} C_{1 V} C_{2 V}=4 g+2 q-4<0$; in particular $g=0$ and $q \leqslant 1$. Therefore (see the Corollary in 3.5) $1 \geqslant q \geqslant e_{1}+e_{2}$ (since $e_{1} \geqslant 0, e_{2} \geqslant 0$ ).

Let $e_{1} \geqslant e_{2}$, and let $e_{1}=1, e_{2}=0$. Then $\left.B\right|_{C_{1}}=\left.2 C_{2}\right|_{C_{1}}$ is not a reducible divisor, which is impossible. Therefore $e_{1}=e_{2}=0$ and $K_{V} \equiv-C_{1 V}-C_{2 V}-F_{V}$. The manifold $V$ is a two-sheeted covering of $S_{1} \times{ }_{C} S_{2}=\left(P^{1} \times P_{o}^{1}\right) \times{ }_{P_{o}^{1}}\left(P_{o}^{1} \times\right.$ $\left.P^{1}\right) \simeq P^{1} \times P^{1} \times P^{1}$ with a branch divisor $B=2 C_{1}+2 C_{2}+2 F$ of multidegree $(2,2,2)$

3.7.2. Let $\operatorname{deg} \pi=1$. Then $V=S_{1} \times{ }_{C} S_{2}$, and $K_{V} \equiv-2 C_{1}-2 C_{2}+$ $\left(2 g-2-e_{1}-e_{2}\right) . F$, where $K_{V} C_{1} C_{2}=2 g-2+e_{1}+e_{2}$ and $K_{V}{ }^{3}=24 .(2 g-2)$. If $V$ is a Fano threefold, then $K_{V}{ }^{3}<0$, hence $g=0$. Therefore $K_{V} C_{1} C_{2}$ $=e_{1}+e_{2}-2<0$, where $e_{1} \geqslant 0, e_{2} \geqslant 0$.

Let $e_{1} \geqslant e_{2}$, and let, for example, $e_{1}=1, e_{2}=0$. Then $V=\mathbf{F}_{1} \times{ }_{P^{1}}\left(P^{1} \times P^{1}\right)$, and $K_{V} \equiv-2 C_{1}-2 C_{2}-3 F, K_{V} C_{1} C_{2}=-1, K_{V}{ }^{3}=-48, K_{V} \sigma_{1}=K_{V} \sigma_{2}=-2$ On the other hand, $-\left.K_{V}\right|_{F}=2 \sigma_{1}+2 \sigma_{2},-\left.K_{V}\right|_{C_{1}}=\left.2 C_{2}\right|_{c_{1}}+\varphi_{1}$, and $-\left.K_{V}\right|_{C_{2}}$ $=\left.2 C_{1}\right|_{C_{2}}+3 \varphi_{2}$ are ample divisors on the surfaces $F \simeq P^{1} \times P^{1}, C_{1} \simeq P^{1} \times P^{1}$, and $C_{2} \simeq \mathbf{F}_{1}$. Since $K_{V} . C_{12}=-1$, and $C_{12}=C_{1} \cap C_{2}$ is a rational curve, then there exists an extremal ray $R_{3}=\mathbf{R}_{+}\left[C_{12}\right]$ (see [3]). As $\left(\left.C_{2}\right|_{C_{1}},\left.C_{2}\right|_{C_{C}}\right)=0$, then $\left.C_{2}\right|_{C_{1}}$ moves in $C_{1}$ as one of the rulings of the quadric $D=C_{1} \simeq P^{1} \times P^{1}$. The restriction of the normal bundle $N_{C_{1} \mid V}$ on $C_{12}$ has a degree -1 . In fact $\left.N_{C_{1} \mid V}\right|_{C_{12}} \simeq$ $\mathscr{O}_{C_{1}}\left(C_{1}\right) \otimes \mathscr{O}_{C_{12}} \simeq \mathscr{O}_{C_{1}}\left(-\sigma_{1}\right) \otimes \mathscr{O}_{C_{12}} \simeq \mathscr{O}_{C_{12}}(-1)$, where $C_{12}=P^{1}$. Therefore, we can contract $C_{1}$ along $C_{12}$ (see [2, p.1020], or [3]); that is, there is an extremal ray of type $E_{1}$ on $V$, i.e. $V$ is not strongly primitive (see 1.4 and 0.2 .2 ).

Let $e_{1}=e_{2}=0$. Then $V \simeq\left(P^{1} \times P_{o}^{1}\right) \times_{P_{o}^{1}}\left(P_{o}^{1} \times P^{1}\right) \simeq P^{1} \times P^{1} \times P^{1}$, and $-K_{V}=2 C_{1}+2 C_{2}+2 F$ is a divisor of multidegree $(2,2,2)$ in $P^{1} \times P^{1} \times P^{1}$.

\subsection{The discriminant curves for $\pi_{1}$ and $\pi_{2}$}

Let $\Delta_{1}$ and $\Delta_{2}$ be the discriminant curves of $\pi_{1}$ and $\pi_{2}$. Then $\Delta_{1} \equiv 4 b_{1}+$ $\left(4 q-2 e_{2}\right) \varphi_{1}$ on $S_{1}$, and $\Delta_{2} \equiv 4 b_{2}+\left(4 q-2 e_{1}\right) \varphi_{2}$ on $S_{2}$. These numerical equalities follow immediately from the formula $-4 . K_{S} \equiv \pi_{*}\left(-K_{V}\right)^{2}+\Delta$, connecting the discriminant curve $\Delta$ of a conic bundle $\pi: V \rightarrow S$ with the canonical bundles 
of $V$ and $S$.

\section{\$4. The Case $(C, D)$}

4.1. Let the extremal morphism $\pi_{1}$ be of the type $C$, and let $\pi_{2}$ be of the type $D$. In particular $\rho(V)=2$ (see 1.3.3). In just the same way as in 2.6 we obtain that $V$ is a Fano threefold.

4.2. Corollary. Let the pair $\left(\pi_{1}, \pi_{2}\right)$ be of the type $(C, D)$. Then $V$ is one of the following (see [4]) :

(1) $V=P^{2} \times P^{1}$;

(2) a two-sheeted covering $\pi: V \rightarrow P^{2} \times P^{1}$ with a branch divisor $B \subset P^{2} \times P^{1}$ of bidegree $(4,2)$;

(3) a two-sheeted covering $\pi: V \rightarrow P^{2} \times P^{1}$ with a branch divisor $B \subset P^{2} \times P^{1}$ of bidegree $(2,2)$.

\section{§5. The Case $(C, E)$}

Let $\pi_{1}$ be of type $C$, and $\pi_{2}$ be of type $E$. We have to prove that if $V$ is strongly primitive, then $V$ must be a Fano threefold. We shall consider separately the cases $E_{2}, E_{3}, E_{4}$, and $E_{5}$ (see 1.3 .1 and 1.4).

5.1. The cases $\left(C, E_{2}\right)$ and $\left(C, E_{5}\right)$

Let $\pi_{2}$ belongs to one of the types $E_{2}$ or $E_{5}$. In particular, the morphism $\pi_{2}$ is a contraction of a divisor $D \simeq P^{2}$ in $V$ to a point (see 1.3.1). The morphism $\pi_{1}$ maps $D \simeq P^{2}$ onto $S_{1}$. Actually, in the opposite case $\pi_{1}$ contracts $D$, because $\pi_{2}(=$ a contracting of $D)$ is an extremal morphism. But $\pi_{1}$ is also an extremal morphism, hence $\pi_{1}$ coincides with $\pi_{2}$ - a cootradiction. Therefore $\pi_{2}(D)=S_{1}$ and $S_{1} \simeq P^{2}$.

\subsection{The cases $\left(C, E_{3}\right)$ and $\left(C, E_{4}\right)$}

Let $\pi_{2}$ belongs to one of the types $E_{3}$ or $E_{4}$. Just as above, the fact that $\pi_{1}$ and $\pi_{2}$ are different extremal morphisms, implies that the morphism $\pi_{1}$ maps the quadric $D \subset V$, corresponding to $\pi_{2}$ (see 1.3.1), onto the surface $S_{1}$. As $S_{1}$ is smooth, it must be either $P^{2}$ (in the cases $E_{3}$ and $E_{4}$ ) or $P^{1} \times P^{1}$ (in the case $E_{3}$ ).

Let $S_{1} \simeq P^{1} \times P^{1}$. Let $\phi_{1}$ and $\phi_{2}$ be the rulings of $S_{1}$, and let $\varphi_{1}=s \times P^{1} \equiv$ $P^{1} \times t=\varphi_{2}$ be the rulings of $D \simeq P^{1} \times P^{1}$. Since $\left(\left.\pi_{1}\right|_{D} ^{*} \phi_{i},\left.\pi_{1}\right|_{D} ^{*} \phi_{i}\right)_{D}=0, i=$ 1,2 , then $\left.\pi_{1}\right|_{D} ^{*} \phi_{i}=m_{i} \varphi_{i}$, where $m_{i}$ is a positive integer. Therefore $\left(\left.\pi_{1}\right|_{D} ^{*} \phi_{1}\right.$, $\left.\left.\pi_{1}\right|_{D} ^{*} \phi_{2}\right)_{D}=\left(m_{1} \varphi_{1}, m_{2} \varphi_{2}\right)_{D}=m_{1} m_{2}$. On the other hand, the last equals to $m=$ $\operatorname{deg}\left(\left.\pi\right|_{D}\right)$. But $\varphi_{1}$ and $\varphi_{2}$ are numerically equivalent on $V$; therefore $0=\varphi_{1} \cdot \pi_{1}^{*} \phi_{1}$ 
$=\varphi_{2} \cdot \pi_{1}^{*} \phi_{1}=m_{2}$. In particular, $\operatorname{deg} \pi=m=m_{1} m_{2}=0$, which is impossible. Consequently, in the cases $\left(C, E_{3}\right)$ and $\left(C, E_{4}\right)$ the surface $S_{1}$ is isomorphic to $P^{2}$.

5.3. Corollary. Let $\pi_{1}$ be of the type $C$ and $\pi_{2}$ be of the type $E\left(E_{2}, E_{3}, E_{4}\right.$, or $\left.E_{5}\right)$. Then $V$ is a Fano threefold.

Proof. In fact, we obtained that in all cases $S_{1} \simeq P^{2}$ (see 5.1 and 5.2). Therefore $\rho(V)=\rho\left(P^{2}\right)+1=2$, and $V$ admits two different extremal morphisms. It follows that $V$ is a Fano threefold (see 2.6).

5.4. Corollary (see [4]). Let $V, \pi_{1}, \pi_{2}$, etc., be as in 5.3. Then $V$ is one of the following:

(1) $V=P\left(\mathscr{O}_{P^{2}} \oplus \mathscr{O}_{P^{2}}(1)\right)$, in the case $\left(C, E_{2}\right)$;

(2) $V=P\left(\mathscr{O}_{P^{2}} \oplus \mathscr{O}_{P^{2}}(2)\right)$, in the case $\left(C, E_{5}\right)$;

(3) $V$ is a two-sheeted covering of $Y=P\left(\mathscr{O}_{P^{2}} \oplus \mathscr{O}_{P^{2}}(1)\right)$ with a branch divisor $B \in\left|-K_{Y}\right|$, in the cases $\left(C, E_{3}\right)$ and $\left(C, E_{4}\right)$.

\section{§6. The case $(D, D)$}

6.1. Let $\pi_{1}$ and $\pi_{2}$ be both of the type $D$ (see 1.3.3). Let $S_{1}=\pi_{1}^{*}(x), x \in$ $C_{1}$, and $S_{2}=\pi_{2}^{*}(x), x \in C_{2}$, where $\pi_{k}: V \rightarrow C_{k}, k=1,2$, are the corresponding extremal morphisms. As $\rho(V)=\rho\left(C_{k}\right)+1=2$, then $S_{1}$ is represented in the form $S_{1} \equiv a . S_{2}+b . K_{V}$, for some rational $a$, $b$. In particular, $K_{S_{1}}=\left(K_{V}+S_{1}\right)$ $\left.\right|_{S_{1}}=\left.(1 / b) \cdot\left(-a \cdot S_{2}+(b+1) \cdot S_{1}\right)\right|_{S_{1}}$. Hence

$$
K_{S_{1}} \cdot K_{S_{1}}=\left(1 / b^{2}\right) \cdot\left((b+1) \cdot S_{1}-a \cdot S_{2}\right)^{2} \cdot S_{1}=0,
$$

since $S_{1} \cdot S_{1}=S_{2} \cdot S_{2}=0$. On the other hand, the divisor $-K_{S_{1}}$ must be ample, since $S_{1}$ is a Del Pezzo surface, $P^{2}$, or $P^{1} \times P^{1}$ (see 1.3.3). We come to a contradiction.

6.2. Corollary. There are no manifolds for which $\pi_{1}$ and $\pi_{2}$ are both of type $D$.

§7. The case $(E, E, \ldots, E)$

Let $V$ admits morphisms $\pi_{1}, \pi_{2}, \ldots, \pi_{n}$ of the type $E$, and let $V$ be strongly primitive. Let $D_{1}, D_{2}, \ldots, D_{n}$ be the corresponding divisors on $V$, which $\pi_{1}, \pi_{2}, \ldots, \pi_{n}$ 
contract (see 1.3.1). Then, by [4, p. $124(8.1)]$, the divisors $D_{i}$ are mutually disjoint. Consequently, the contractions $\pi_{i}$ carry out independently.

Theorem 1.5 is proved.

\section{REFERENCES}

[1] R. Hartshorne, Algebraic Geometry, Springer-Verlag (1977).

[2] V.S. Kulikov, Degenerations of $K 3$-surfaces and Enriques surfaces, Math. USSR Izvestija, Vol. 41, No. 5 (1977), 1008-1042 (in Russian).

[ 3 ] S. Mori, Threefolds whose canonical bundles are not numerically effective, Ann. of Math., 116 (1982), 133-176.

[4] S. Mori, S. Mukai, On Fano 3 -folds with $B_{2} \geqslant 2$, Adv. St. in Pure Math., Vol.1-Algebraic Varieties and Analytic Varieties, Kinokuniya Comp. LTD (1983), $101-129$.

[5] E. Sato, Varieties which have two projective space bundle structures, J. Math. Kyoto Univ., 25 (1985), 445-457.

Institute of Mathematics

Bulgarian Academy of Sciences

ul. Acad. G. Bonchev, bl.8

1113 Sofia, Bulgaria 